\title{
ON H"-CLOSED SETS IN TOPOLOGICAL SPACES
}

\author{
${ }^{1}$ A. JOSE LITTLE FLOWER AND ${ }^{2}$ M. RAJA KALAIVANAN \\ ${ }^{1}$ Research Scholar, Department of Mathematics, \\ Madurai Kamaraj University, Madurai, Tamil Nadu, India -625 021. \\ e-mail : joselittlef@gmail.com. \\ ${ }^{2}$ Department of Mathematics, \\ Pasumpon Muthuramalinga Thevar College, \\ Usilampatti - 625 532, Madurai District, Tamil Nadu, India. \\ e-mail : rajakalaivanan@yahoo.com
}

\begin{abstract}
In this article, we introduce a new class of closed sets in topological spaces namely, $\mathrm{H}^{\prime \prime}$-closed and we prove every subset of the digital line is $\mathrm{H}^{\prime \prime}$-closed.
\end{abstract}

Keywords: $H^{\prime \prime}$-closed sts, $H^{\prime \prime}$-open sets $\Lambda_{-s e t s}$ and $\Lambda_{r}-$ sets.

\section{INTRODUCTION}

J. Jeyanthi et.al the introduced $\Lambda_{r}$-closed, $\Lambda_{r}$ continuous and Caldas et.al the introduced $\lambda$ closed and $\lambda$-continuous. Levin introduced generalized closed sets developed by more generalized sets.

In this paper, we introduce a new class of closed sets in topological spaces namely, $\mathrm{H}^{\prime \prime}$-closed and we prove every subset of the digital line is $\mathrm{H}^{\prime \prime}$ closed.

Through out paper obtained in the Topological space $(X, \tau)$ (resp. $(X, \sigma)$ and $(X, \eta))$ is denoted by TS $X$ (resp. TS Y and TS Z).

For a subset $\mathrm{C}$ of a TS $\mathrm{X}, \operatorname{int}(\mathrm{C}), \operatorname{cl}(\mathrm{C})$ denoted the interior, closure of $C$ respectively. And $\lambda$ symbol use this thesis A.

For so many author introduced various definitions

Definition 1.1. [5] The collection of all $\lambda$ closed(resp. $\lambda$-open) subsets of $X$ will be denoted by $\lambda C(X)($ resp. $\lambda O(X))$. We set

$\lambda C(X, X)=\{U: x \in O \in \lambda C(X, \tau)\} \lambda O(X, X)=\{U: x \in$ $O \in \lambda O(X, \tau)\}$

Definition 1.2. [8] Let $B$ be a subset of a TS $X$. We define subsets $B^{\Lambda}$ and $B^{V}$ as follows:

$$
\begin{aligned}
B^{\Lambda}=\cap\{U / U \supseteq B, U & \in \tau\}, \text { and } \\
B^{V} & =\cup\{F / F \subseteq B, X-F \in
\end{aligned}
$$

$\tau\}$

$A$ subset $B$ of $(X, \tau)$ is a $\Lambda$-set (resp. $V$-set) if $B=B^{\Lambda}$ (resp. $B=B^{V}$ ).

Definition 1.3. A subset $C$ of a TS $X$ is called a

(1) $g$-closed set [7] if $C l(C) \subseteq U$ whenever $C \subseteq U$ and $U$ is open.

The complement of g-closed set is g-open set.

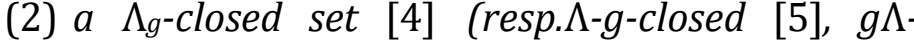
closed) if $\mathrm{Cl}(C) \subseteq U$ (resp. $\left.\mathrm{Cl} \lambda(C) \subseteq U, C l_{\lambda}(C) \subseteq U\right)$ whenever $C \subseteq U$ and $U$ is $\lambda$-open (resp. $U$ is $\lambda$ open, $U$ is open).

Lemma 1.4. [2] Let $C$ be a subset of a TS X. Then we have the next:

(1) If $C \subset X$ then $C \subset \lambda \operatorname{ker}(C)$.

(2) If $C, B \subset X$ then $C \subset B$ implies $\lambda \operatorname{Ker}(C) \subset \lambda \operatorname{Ker}(B)$.

(3) $\lambda \operatorname{Ker}(\lambda \operatorname{Ker}(C))=\lambda \operatorname{Ker}(C)$.

Proposition 1.5. [8]

(1) The subsets $\varphi$ and $X$ are $\Lambda$-sets.

(2) Every union of $\Lambda$-sets is a $\Lambda$-set.

(3) Every intersection of $\Lambda$-sets is a $\Lambda$-set.

(4) $A$ subset $B$ is a $\Lambda$-set if and only if the complement of $B$ is a $V$-set.

Lemma 1.6. [9] Every $\Lambda_{r}$-set is $\Lambda$-set.

$\mathrm{H}^{\prime \prime}$-sets and $\mathrm{K}^{\prime \prime}$-sets

This section contains a new class of sets, called $\mathrm{H}^{\prime \prime}$-sets in TS and investigate certain basic properties of $\mathrm{H}^{\prime \prime}$-sets.

Definition 2.1. Let $S$ be a subset of a TS $X$, then we define a $S S^{\prime \prime}=\cap\{Q / Q \supset S, Q \in A O(X, \tau)\}$.

Lemma 2.2. [5] Let $C, B$ and $C_{i}(i \in I)$ be a subset of a TS X. The following properties hold:

(1) $C \subset \operatorname{Acl}(C) \subset \operatorname{cl}(C)$.

(2) $C \subset B \Rightarrow \operatorname{Acl}(C) \subset \operatorname{Acl}(B)$.

(3) $C$ is A-closed $\Leftarrow \Rightarrow C=\operatorname{Acl}(C)$.

(4) $\operatorname{Acl}(\operatorname{Acl}(C))=\operatorname{Acl}(C)$. (5) If $C_{i}$ is A-closed for each $i \in I$ then $\cap C_{i}$ is A-closed.

\section{$i \in I$}

(6) If $C_{i}$ is A-open for each $i \in I$ then $\cup C_{i}$ is Aopen.

$i \in I$

Lemma 2.3. For the subsets $C, B$ and $C_{i}(i \in I)$ of a TS $X$ the following hold.

(1) If $C \in \mathrm{A} O(X, \tau)$ then $C=C S^{\prime \prime}$. 
(2) $\left(\cup C_{i}\right) \mathrm{S}^{\prime \prime}=\cup C_{i} \mathrm{~S}^{\prime \prime}$.

$$
i \in I \quad \text { " } i \in I \text { " }
$$

(3) $\left(\cap C_{i}\right) \mathrm{S} \subset \cap C_{i}$ S. $i \in I \quad i \in I$

roof. (1) By Definition 2.1 and since $C \in \mathrm{A} O(X, \tau)$, we have $C S^{\prime \prime} \subseteq C$. By Lemma 1.4(1), we have that $C$ $=C S^{\prime \prime}$.

(2) Assume that there exists a point $x \in X$ such that $x / \in \cup C_{i}$ s. Then by $i \in I$ Definition 2.1, there exists subsets $W_{i} \in \mathrm{A} O(X, \tau)$, for all $i \in I$, such that $x / \in W_{i}, C_{i} \subset W_{i}$. Let ${ }^{W}=\cup_{i \in I} P_{i}$. Then we have that $x \notin \cup_{i \in I} W_{i}, \cup_{i \in I} C_{i} \subseteq W$ and $W \in \mathrm{A} O(X, \tau)$. This implies that $x \notin\left(\cup_{i \in I} C_{i}\right)^{\mathscr{\mathcal { S }}}$. Thus ( $\left.\cup_{i \in I} C_{i}\right)^{\mathcal{S}^{\prime \prime}} \subset \cup_{i \in I} C_{i}$ s $^{\prime \prime}$.

Conversely, Assume that there exists a point $x$ such that $x \notin\left(\cup_{i \in I} C_{i}\right)^{\mathcal{S}}$. Then there exists a subset $W \in \mathrm{A} O(X, \tau)$ such that $\cup C_{i} \subset W$ and $x / \in W$.

Thus, for each $i \in I$ we have $x / \in C_{i}$ s. This implies that $x \notin \cup \cup_{i \in I}\left(C_{i}^{\mathcal{S}}\right)$. Thus, $\cup_{i \in I} C_{i}^{\ddot{\mathcal{S}}} \subset\left(\cup_{i \in I} C_{i}\right)^{\ddot{\mathcal{S}}}$.

(3) Suppose that there exist a point $x$ such that $x / \epsilon$ $\cap C_{i} S^{\prime \prime}$. Then there exists

$\in I$ " $i_{0} \in I$ such that $x / \in\left(C_{i 0}\right)$ Sand there exists Aopen set $P$ such that $x / \in P$ and $C_{i 0} \subset P$. We have $\cap C_{i} \subset C_{i 0} \subset P$ and $x / \in P$. Therefore, $x / \in(\cap$ $\left.C_{i}\right) \mathrm{S}^{\prime \prime} . i \in I \quad i \in I$ This shows that $\left(\bigcap_{i \in I} C_{i}\right)^{\ddot{\mathcal{S}}} \subset \bigcap_{i \in I} C_{i}^{\mathcal{S}}$.

Remark 2.4. The converse of above Theorem 2.3

(1) is not true and the equality of

(3) is not always true in general.

Example 2.5. Let $X=\{1,2,3,4\}$ with $\tau=\{X, \varphi,\{1$, $3\},\{1,3,4\}\}$.

By Definition 2.1, $(\{4\}) \mathrm{S}^{\prime \prime}=\{4\}$, the set $\{4\}$ is not A-open.

Definition 2.6. $A$ subset $C$ of a TS $X$ is called $\mathrm{S}^{\prime \prime}$ set if $C=C S^{\prime \prime}$.

The family of all $\mathrm{H}^{\prime \prime}$-sets of TS $X$ is denoted by $\tau \mathrm{S}^{\prime \prime}(X)$ (or simply $\left.\tau \mathrm{S}^{\prime \prime}\right)$.

Proposition 2.7. In a TS $X$, every $\Lambda$-set is $\mathrm{S}^{\prime \prime}$-set. Proof. Follows from the fact that every open set is A-open.
Example 2.8. Let $X=\{1,2,3\}$ and $\tau=\{\varphi, X,\{1\}$, $\{1, b\}\}$. Hence, $\{2\}$ is a $\mathrm{S}^{\prime \prime}$-set but not a $\Lambda$-set.

Definition 2.9. Let $Q$ be a subset of a TS $X$ then we define the following:

$Q \mathrm{~K}^{\prime \prime}=\cap\{P / P \subset Q, P \in \mathrm{A} C(X, \tau)\}$

Definition 2.10. A subset $C$ of a TS $X$ is called $\mathrm{K}^{\prime \prime}$ set if $C=C \mathrm{~K}^{\prime \prime}$.

The family of all $\mathrm{K}^{\prime \prime}$-sets of TS $\mathrm{X}$ is denoted by $\tau \mathrm{K}^{\prime \prime}(X)$ (or simply $\tau \mathrm{K}^{\prime \prime}$ ).

Lemma 2.11. For subsets $C$ and $C_{i}(i \in I)$ of a TS $X$, the following hold.

(1) The subsets $\varphi$ and $X$ are $S^{\prime \prime}$-sets.

(2) If C is A-open then $C$ is a $\mathrm{S}^{\prime \prime}$-set.

(3) If $C_{i}$ is a $\mathrm{S}^{\prime \prime}$-set for each $i \in I$ then $\cup_{i \in I} C_{i}$ is a S"-set. (4) If $C_{i}$ is a $\mathrm{S}^{\prime \prime}$-set for each $i \in I$ then $\cap_{i \in I} C_{i}$ is a $\mathrm{S}^{\prime \prime}$ set.

Proof. (1) Follows from Proposition 1.5(1) and Proposition 2.7.

(2) Follows from Lemma 2.3(1) and Definition 2.6.

(3) Let $C_{i} \in \tau S^{\prime \prime}$ for some $i \in I$, then we have, $\cup C_{i \in I} C_{i}=\cup \cup_{i \in \mathcal{G}} C_{i}^{\mathcal{S}}$ by Definition $2.6 \cup C_{i}=\cup C_{i} S^{\prime \prime}=(\mathrm{U}$ $\left.C_{i}\right) \mathrm{S}^{\prime \prime}$ by Lemma 2.3, $\cup C_{i}=\cup C_{i} \mathrm{~S}^{\prime \prime}=\left(\cup C_{i}\right) \mathrm{S}^{\prime \prime} \supset \cup C_{i}$ by $i \in I \quad i \in I \quad i \in I \quad i \in I^{\prime} i \in I i \in I$ " $i \in I$

Lemma 1.4(1). Thus, we have $\cup C_{i}=\left(\cup C_{i}\right) \mathrm{S}$. Therefore $\cup C_{i} \in \tau \mathrm{S}$.

$i \in I \quad i \in I$ $i \in I$

(4) Let $C_{i} \in \tau S$ for each $i \in I$, then by Definition 2.6, Lemma 2.3 and 1.4(1) we have, $\bigcap_{i \in I} C_{i}=\bigcap_{i \in I} C_{i}^{\mathcal{S}} \supset\left(\bigcap_{i \in I} C_{i}\right)^{\mathcal{S}} \supset \bigcap_{i \in I} C_{i}$. Thus, we have $\bigcap_{i \in I} C_{i}=\left(\bigcap_{i \in I} C_{i}\right)^{\mathcal{S}}$ and

$\cap_{i \in I} C_{i} \in \tau^{\mathcal{\mathcal { S }}}$.

Theorem 2.12. In a TS $X$, if $\mathrm{A} O(X, \tau)=\mathrm{AC}(X, \tau)$ then for any subset $C \subset X, \operatorname{Acl}(C)=C S^{\prime \prime}$.

Proof. Let $C \subset X$ and $C \in \mathrm{A} O(X, \tau)$. By Lemma 2.3(1) we have, $C S^{\prime \prime}=C$. By assumption, $C \in$ $\mathrm{A} C(X, \tau)$. Then, by Lemma 2.2(3) $\operatorname{Acl}(C)=C$. Therefore $\operatorname{Acl}(C)=C S^{\prime \prime}$.

Remark 2.13. The converse of Theorem 2.12 is not true in general.

Example 2.14. Let $X=\{1,2,3,4,5\}$ and $\tau=\{\varphi, X$, $\{3,4\},\{1,3,4\}\}$. Let $C=\{1\}$. Then $\operatorname{Acl}(C)=\{1\}$ and $A S^{\prime \prime}=\{1\}$ but $\mathrm{A} O(X, \tau) 6=\mathrm{AC}(X, \tau)$.

\section{$\mathbf{H}^{\prime \prime}$-closed sets}

We introduce a new class of sets, called $\mathrm{H}^{\prime \prime}$ closed sets in space and study 
their properties.

Definition 3.1. A subset $C$ of a TS $X$ is called $\mathrm{H}^{\prime \prime}$ closed if $C=P \cap Q$ where $P$ is a $\mathrm{H}^{\prime \prime}$-set and $Q$ is a A-closed set.

The complement of $\mathrm{H}^{\prime \prime}$-closed set is called $\mathrm{H}^{\prime \prime}$ open set.

Lemma 3.2. Let a TS $X$. Then the following properties are valid:

(1) $\varphi$ and $X$ are A-closed and A-open in $X$.

(2) A-closed set in $X$ is $\mathrm{H}^{\prime \prime}$-closed in $X$.

(3) $\varphi$ and $X$ are $\mathrm{H}^{\prime \prime}$-closed and $\mathrm{H}^{\prime \prime}$-open in $X$.

Proof. (1) Since $\varphi$ can be written as $\varphi \cap X, X$ is closed in TS X and by Proposition 1.5(1), $\varphi$ is a $\Lambda$-set we have $\varphi$ is a A-closed set in TS X. Since $X=X \cap X, \mathrm{X}$ is closed in TS $\mathrm{X}$ and by the Proposition 1.5, $\mathrm{X}$ is a $\Lambda$-set, $\mathrm{X}$ is $\mathrm{A}$-closed in $\mathrm{X}$. The complement of $\varphi$ and $\mathrm{X}$ is $\mathrm{X}$ and $\varphi$ respectively. Hence $\varphi$ and $\mathrm{X}$ are A-open in X.

(2) Let A be a A-closed set in X. X is A-open. By Lemma 2.11 (2), $\mathrm{X}$ is a $\mathrm{S}^{\prime \prime}$-set in X. Hence $C=C \cap$ $X$ is a $\mathrm{H}^{\prime \prime}$-closed set in $\mathrm{X}$.

(3) By (1) and (2), we get $\varphi$ and $X$ are $H^{\prime \prime}$-closed. The complement of $\varphi$ and $\mathrm{X}$ is $\mathrm{X}$ and $\varphi$ respectively. Hence $\varphi$ and $\mathrm{X}$ are $\mathrm{H}^{\prime \prime}$-open in $\mathrm{X}$.

Remark 3.3. In general the converse of (2) of Lemma 3.2 is not true which is seen in the next Example.

Example 3.4. Let a TS $X$ such that $X=\{1,2,3,4\}$ and $\tau=\{\varphi,\{2\},\{1,2\},\{2,3\},\{1,2,3\}, X\}$. Hence $\{1,2,4\}$ is $\mathrm{H}^{\prime \prime}$-closed but not A-closed.

Theorem 3.5. In a TS X, every $\Lambda_{r}$-closed is $\mathrm{H}^{\prime \prime}$ closed.

Proof. Suppose $C=P \cap Q$ is $\Lambda_{r}$-closed, where $\mathrm{P}$ is a $\Lambda_{r}$-set and Q is closed. By Lemma $1.6, \mathrm{P}$ is a $\Lambda$ set. Thus $\mathrm{C}$ is $\mathrm{A}$-closed and hence $\mathrm{C}$ is $\mathrm{H}^{\prime \prime}$-closed.

Example 3.6. Let $X=\{1,2,3,4\}, \tau=\{\varphi, X,\{1, b\}$, $\{1,2,3\}\}$. Hence $\{3\}$ is not

a $\Lambda$-closed set but it is a $\mathrm{H}^{\prime \prime}$-closed set.

Theorem 3.7. In a TS $X$, every $(\Lambda, \theta)$-closed is $(\Lambda, \delta)$-closed.

Proof. Follows from the fact that every $\theta$-closed is $\delta$-closed and $\theta$-open is $\delta$-open.

Example 3.8. Let $X=\{1,2,3,4\}, \tau=\{\varphi, X,\{1\},\{2\}$, $\{1,2\}\}$. Hence $\{1\}$ is a

$(\Lambda, \delta)$-closed set but not a $(\Lambda, \theta)$-closed set.

Lemma 3.9. In a TS $X$, every $\Lambda_{\theta}$-set is $\Lambda$-set.

Proof. Follows from the fact that every $\theta$-open is open.

Example 3.10. Let $X=\{1,2,3\}, \tau=\{\varphi, X,\{1\},\{1$, $2\}$. Hence $\{1\}$ is a $\Lambda$-set but not a $\Lambda_{\theta}$-set.
Theorem 3.11. In a TS $X$, every $(\Lambda, \theta)$-closed is $\mathrm{H}^{\prime \prime}$-closed.

Proof. Suppose $C=P \cap Q$ is $\Lambda_{\theta}$-closed, where $\mathrm{P}$ is a $\Lambda_{\theta}$-set and Q is $\theta$-closed. By Lemma 3.9, $\mathrm{L}$ is a $\Lambda$-set. Since every $\theta$-closed set is a closed set, $\mathrm{Q}$ is closed. Thus $\mathrm{C}$ is A-closed and hence $\mathrm{C}$ is $\mathrm{H}^{\prime \prime}$-closed.

Example 3.12. Let $X=\{1,2,3,4\}, \tau=\{\varphi, X,\{1\}$, $\{2\},\{1,2\}\}$. Hence $\{1\}$ is

$\mathrm{H}^{\prime \prime}$-closed but not $(\Lambda, \theta)$-closed.

Remark 3.13.The concepts of closed sets and $(\Lambda, \delta)$-closed sets are independent.

\section{Example 3.14.}

(1) Let $X=\{1,2,3\}, \tau=\{\varphi, X,\{1\},\{1,2\}\}$. Hence $\{3\}$ is closed but not

$(\Lambda, \delta)$-closed.

(2) Let $X=\{1,2,3\}, \tau=\{\varphi, X,\{1\},\{2\},\{1,2\}\}$. Here $\{3\}$ is $(\Lambda, \delta)$-closed but

not closed.

Lemma 3.15. In a TS X, every $\Lambda_{\delta}$-set is $\Lambda$-set.

Proof. Follows from the fact every $\delta$-open is open.

Example 3.16. Let $X=\{1,2,3\}, \tau=\{\varphi, X,\{1\}\}$. Hence $\{1\}$ is a $\Lambda$-set but not a

$\Lambda \delta$-set.

Theorem 3.17. In a TS $X$, every $(\Lambda, \delta)$-closed is $\mathrm{H}^{\prime \prime}$-closed.

Proof. Suppose $C=P \cap Q$ is $\Lambda_{\delta}$-closed, where $\mathrm{P}$ is a $\Lambda \delta$-set and Q is $\delta$-closed. By Lemma 3.15, P is a $\Lambda$-set. Since every $\delta$-closed set is a closed set, $\mathrm{Q}$ is closed. Thus $\mathrm{C}$ is A-closed and hence $\mathrm{C}$ is $\mathrm{H}^{\prime \prime}$ closed.

Example 3.18. Let $X=\{1,2,3\}, \tau=\{\varphi, X,\{1\},\{1$, $2\}\}$. Hence $\{1\}$ is $\mathrm{H}^{\prime \prime}$-closed

but not $(\Lambda, \delta)$-closed.

Theorem 3.19. For a subset $C$ of a TS $X$, the following properties are equivalent.

(1) $C$ is $\mathrm{H}^{\prime \prime}$-closed.

(2) $C=P \cap A c l(C)$, where $P$ is a $\mathrm{H}^{\prime \prime}$-set.

(3) $C=C S^{\prime \prime} \cap \operatorname{Acl}(C)$.

Proof. (1) $\Rightarrow$ (2) : Let $C$ be $\mathrm{H}^{\prime \prime}$-closed, then there exists a $\mathrm{H}^{\prime \prime}$-set $\mathrm{P}$ and a A-closed such that $C=P$ $\cap Q$. Since $C \subset Q$, We have $C \subset \operatorname{Acl}(C) \subset \operatorname{Acl}(Q)=$ $Q$ and $C=P \cap Q \supset P \cap \operatorname{Acl}(C) \supset C$. Therefore we obtain $C=P \cap \operatorname{Acl}(C)$.

(2) $\Rightarrow$ (3) : Let $C=P \cap \operatorname{Acl}(C)$, where $P$ is a $\mathrm{H}^{\prime \prime}$-set. Since $C \subset P$, we have $C S^{\prime \prime} \subset P S^{\prime \prime}=\mathrm{P}$ and hence $C \subset C^{\prime \prime} \cap \operatorname{Acl}(C) \subset P \cap \operatorname{Acl}(C)=C$. Therefore, we obtain $C=C S^{\prime \prime} \cap \operatorname{Acl}(C)$. 
(3) $\Rightarrow(1)$ : By Lemma 1.4, CS" is a $\mathrm{S}^{\prime \prime}$-set and by Lemma 2.2(4), $\mathrm{Acl}(C)$ is A-closed. By (3), $C=$ $C S^{\prime \prime} \cap \mathrm{Acl}(C)$ and hence $\mathrm{C}$ is $\mathrm{H}^{\prime \prime}$-closed.

Theorem 3.20. If a set $C$ is $\Lambda$-g-closed then $\mathrm{Acl}(\mathrm{C}) / C$ contains no non empty

A-closed.

Proof. Let $\mathrm{G}$ be a A-closed subset of $\operatorname{Acl}(A)-C$. Now, we have $C \subseteq X-G$. Since $C$ is $\Lambda$-g-closed, we have $\operatorname{Acl}(C) \subseteq X-G$ (or) $G \subseteq X-(\operatorname{Acl}(C))$. Thus $G \subseteq \operatorname{Acl}(C) \cap(X-(\operatorname{Acl}(C)))=\varphi$ and $\mathrm{G}$ is $\varphi$.

Remark 3.21. The converse of Theorem 3.20 is not true as it can be seen by the next Example.

Example 3.22. Let $X=\{1,2,3,4\}, \tau=\{\varphi, X,\{1\}$, $\{1, b\}\}$. If $C=\{1,3\}$ then $\operatorname{Acl}(C)-C=\{2,4\}$ does not contain nonempty A-closed set. But $C$ is not $\Lambda-g$ closed.

\section{References}

1. F. G. Arenas, Julian Dontchev and Maximilian Ganster, On $\lambda$-sets and the dual of generalized continuity, Questions Answers Gen. Topology 15 (1997), 3-13.

2. M. Caldas, D. N. Georiou and S. Jafari, Characterization of low separation axioms via $\lambda$-open sets and $\lambda$-closure operator, Rendiconti del Circ. Mat. Di Palermo, 54(2005), 195-208.

3. M. Caldas, D. N. Georgiou, S. Jafari and T. Noiri, On $(\Lambda, \theta)$-closed sets, $Q$ and $A$ in General Topology, 23(2005).

4. M. Caldas, S. Jafari and T. Noiri, On $\Lambda$ Generalized closed sets in topological spaces, Acta. Hungar., 118(4)(2008), 337343. 